\title{
Planejamento Estratégico para Qualificação da Programação dos Medicamentos para Cessação do Tabagismo: um relato de experiência
}

\author{
Strategic Planning to Qualify the Programming of Smoking \\ Cessation Medicines: an experience report
}

\section{Planificación Estratégica para la Calificación de la Programación de los Medicamentos para Dejar de Fumar: un informe basado en experiencia}

Deborah Alencar de Oliveira ${ }^{1}$ Janeth de Oliveira Silva Naves ${ }^{2}$

\begin{abstract}
RESUMO: O tratamento para cessação do tabagismo tem reconhecida efetividade e é disponibilizado pelo Sistema Único de Saúde (SUS). Esse estudo teve como objetivo descrever a experiência de planejamento participativo para a qualificação da programação de medicamentos do Programa Nacional de Controle do Tabagismo (PNCT). Trata-se de relato de experiência construído com base no Planejamento Estratégico Situacional, a partir da realização de oficinas com gestores municipais, estaduais e federais da assistência farmacêutica. Foi constatada uma ausência de planejamento estratégico com consequente irregularidade no abastecimento de medicamentos e prejuízo aos tratamentos ofertados pelo PNCT. O planejamento para o problema priorizado de dificuldade na programação dos medicamentos para cessação do tabagismo apontou objetivos, operações e ações, analisou sua viabilidade e factibilidade, e traçou indicadores para a avaliação e o monitoramento de sua implementação. A demanda por medicamentos e o abastecimento da rede puderam ser levantados e a relação com os gestores locais foi fortalecida. A carência de alinhamento e articulação entre as diferentes áreas gestoras do PNCT comprometeu a concretização de algumas das ações do plano, reforçando a necessidade de mobilização de todos gestores quanto à importância da construção conjunta de um planejamento capaz de qualificar a gestão do programa, de forma ampla, proporcionando assim a regularização e ampliação dos tratamentos para cessação do tabagismo pelo SUS.
\end{abstract}

Palavras-chave: Assistência Farmacêutica, Planejamento Estratégico, Abandono do Uso de Tabaco, Programa Nacional de Controle do Tabagismo.

ABSTRACT: The treatment for smoking cessation has a recognized effectiveness and is available in the Brazilian Healthcare System (SUS). The objective of this study was to describe

\footnotetext{
1 Graduada em Ciências Farmacêuticas e Mestre em Neurociências (Psicofarmacologia e Adicção a Drogas) pela Universidade de Brasília, e Especialista em Gestão da Assistência Farmacêutica pela Universidade de Santa Catarina. Consultora Técnica do Ministério da Saúde entre 2013 e 2016, coordenando os serviços de Assistência Farmacêutica do Programa Nacional de Controle do Tabagismo. Ministério da Saúde. Brasília, Distrito Federal, Brasil. E-mail: oliveiradeborah@yahoo.com.br

2 Mestre e Doutora em Ciências da Saúde pela Universidade de Brasília (UnB). Especialista em microbiologia pelo Instituto de Patologia Tropical da Universidade Federal de Goiás. Possui graduação em Farmácia e graduação em Tecnólogo em Saneamento Ambiental pela Universidade Federal de Goiás. Universidade de Brasília (UnB). Brasília, Distrito Federal, Brasil. E-mail: janeth_naves@hotmail.com
}

ISSN 1982-8829 Tempus, actas de saúde colet, Brasília, 11(3), 247-258, 2017 - Epub mar, 2018 
the experience of participatory planning in order to qualify the medicines programming of the National Tobacco Control Program (NTCP). It is an experience report built during workshops with municipal, state and federal managers of pharmaceutical assistance, applying the Situational Strategic Planning method. It was verified a lack of strategic planning with consequent irregularity in the medicines supply, compromising the treatments offered by NTCP. The planning for the prioritized problem of difficulty in programming the NTCP medicines pointed out objectives, operations and actions, analyzed its viability and feasibility, and traced indicators for evaluating and monitoring its implementation. The actual demand for medicines and the supply coverage could be obtained, as well as the relationship between managers was strengthened. The lack of alignment and coordination between the different areas involved in the management of the NTCP compromised achieving some of the plan's actions, reinforcing the need of raising awareness of all managers towards the importance of building together a plan able to broadly qualify the NTCP management, thus allowing regulation and expansion of smoking cessation treatments through SUS.

Keywords: Pharmaceutical Services, Strategic Planning, Tobacco Use Cessation, National Program of Tobacco Control.

RESUMEN: El tratamiento para dejar de fumar tiene eficacia reconocida e es accesible desde el Sistema de Salud Brasileño. Este estudo tiene el objetivo de describir la experiencia de planificación participativa para la calificación de programación de medicamentos del Programa Nacional para el Control del Tabaco (PNCT). Es un informe de experiencia construido a través de workshops con gestores municipales, estatales y federales de la atención farmacéutica, aplicando el método de Planificación Estratégica Situacional. Se constató una falta de planificación, con la consiguiente irregularidad en el suministro de medicamentos, comprometiendo los tratamientos ofrecidos por el PNCT. La planificación para lo problema priorizado de dificultad en programar medicamentos del PNCT señaló los objetivos, operaciones y acciones, analizó su viabilidad y factibilidad, y trazó los indicadores para su evaluación y control. La demanda de los medicamentos y la situación del suministro podrían elevarse y se fortaleció el contacto con los administradores locales. La falta de coordinación entre las áreas de gestión del PNCT compromiso el logro de algunas de las acciones del plan, lo que refuerza la necesidad de mobilizar todos los directivos sobre la importancia de construir conjuntamente una planificación capaz de ampliamente calificar la gestión del PNCT, proporcionando así la regularización y ampliación de los tratamientos para cesación del tabaquismo por el SUS.

Palabras-clave: Servicios Farmacéuticos, Planificación Estratégica, Cese del Uso de Tabaco, Programa Nacional de Control del Tabaquismo.

\section{INTRODUÇÃO}

A Organização Mundial da Saúde(OMS) reconhece o hábito de fumar e de consumir produtos derivados do tabaco como uma doença crônica resultante da dependência à nicotina1. Também considerado um dos principais fatores de risco para as Doenças Crônicas Não-Transmissíveis, como cânceres, doenças cardiovasculares e do aparelho respiratório, estima-se que o tabagismo cause cerca de seis milhões de mortes a cada ano, seja em função do consumo do tabaco ou do fumo passivo2,3. Trata-se, portanto, de uma das principais causas de morbimortalidade prevenível e de um grave problema de saúde pública global2.

O tabagismo também reduz a produtividade da população em função de absenteísmo,

ISSN 1982-8829 Tempus, actas de saúde colet, Brasília, 11(3), 247-258, 2017 - Epub mar, 2018 
invalidez e morte prematura2,3. Um estudo sobre a carga para o Brasil das doenças relacionadas ao tabaco identificou sua relação com $13 \%$ do total de mortes no país em 2008 , estando associadas também a uma redução de cinco anos na expectativa de vida do fumante brasileiro em relação à do não-fumante. Essas doenças acabaram impondo ao país um custo de R $\$ 20,68$ bilhões, somente com assistência direta à saúde4.

A dimensão dos danos associados ao tabagismo obriga os sistemas nacionais de saúde de todo o mundo a manterem seu controle prioritário. A Convenção-Quadro para o Controle do Tabaco da OMS é considerada o marco regulatório dessas medidas de controle e tem o Brasil como signatário desde 20052,5. A gestão do controle do tabaco no país é articulada pelo Ministério da Saúde (MS) através do Programa Nacional de Controle do Tabagismo (PNCT), coordenado pelo Instituto Nacional do Câncer (INCA)5.

O PNCT tem como objetivo proteger a população brasileira das devastadoras consequências sanitárias, sociais, econômicas e ambientais geradas pelo consumo do tabaco5. Para isso, o programa desenvolve ações como o aumento de impostos e advertências sobre o produto, proibição de publicidade, restrição de fumo em ambientes fechados, além da oferta de tratamento para cessação do tabagismo5,6. O efeito dessas medidas tem se revelado em pesquisas nacionais, que demonstram uma redução progressiva do tabagismo nas últimas décadas. Em 1989, a prevalência de fumantes com 18 anos ou mais no Brasil era de 34,8\%, reduzindo para 17,2\% em 2008 e para $14,7 \%$ em $20136,7,8$.

A articulação das ações do PNCT ocorre dentro da rede do Sistema Único de Saúde (SUS), envolvendo equipes nas Secretarias Estaduais de Saúde (SES), que coordenam equipes nas Secretarias Municipais de Saúde (SMS), que, por sua vez, coordenam a execução dessas ações em equipes de saúde geralmente multidisciplinares, presentes em unidades básicas de saúde, ambulatórios, hospitais e universidades5.

A oferta de tratamento para cessação do tabagismo está entre as intervenções com melhor custo-efetividade nos cuidados em saúde9. Isso pois aumenta significativamente as chances do usuário de tabaco alcançar abstinência definitiva à nicotina, elevando seu bem-estar e reduzindo o risco de desenvolver ou agravar problemas de saúde. A combinação de intervenções comportamentais com o uso de medicamentos demonstrou aumentar significativamente as taxas de cessação, chegando a triplicar a abstinência à nicotina, quando comparada a qualquer tratamento isolado9.

O Protocolo Clínico e Diretrizes Terapêuticas (PCDT) da Dependência à Nicotina considera a abordagem cognitivo-comportamental como eixo central do tratamento 10. O apoio medicamentoso deve ser usado sempre em associação com essa abordagem e indicado somente para grau elevado de dependência, uma vez que visa minimizar os sintomas da síndrome de abstinência à nicotina9,10. O SUS disponibiliza os seguintes medicamentos como apoio à abordagem do tabagista11: nicotina 
- nas formas de adesivos transdérmicos ( $7 \mathrm{mg}, 14 \mathrm{mg}$ e $21 \mathrm{mg}$ ), goma de mascar (2mg) e pastilha (2mg) - e cloridrato de bupropiona (150mg).

Pesquisas abrangentes têm revelado que o tratamento para cessação do tabagismo disponibilizado pelos serviços de saúde brasileiros ainda é insuficiente, mesmo frente ao elevado número de tabagistas que desejam ou tentam parar de fumar. Na Pesquisa Nacional de Saúde de $2013,51,1 \%$ dos tabagistas entrevistados afirmaram que tentaram parar de fumar no ano anterior, mas desses somente $8,8 \%$ chegaram a procurar tratamento com profissional de saúde8. A Pesquisa Especial de Tabagismo de 2008 revelou ainda que somente 57,1\% dos entrevistados foram advertidos a parar de fumar no ano anterior à pesquisa e, entre os que conseguiram parar, apenas $15,2 \%$ receberam acompanhamento profissional e $6,7 \%$ usaram medicamentos durante a tentativa de cessação7.

Em resposta à problemática de baixo acesso ao tratamento, o Ministério da Saúde publicou, em 2013, portaria que atualizava as diretrizes de cuidado à pessoa tabagista no SUS e promovia a ampliação da Rede Nacional de Tratamento do Tabagismo (RNTT)12. Utilizando o Programa Nacional de Melhoria do Acesso e da Qualidade da Atenção Básica (PMAQ) como alternativa de acesso, 23.387 novas equipes de saúde aderiram ao PNCT, devendo, em tese, passar a ofertar tratamento para cessação do tabagismo.

Essa normativa manteve a aquisição dos medicamentos centralizada no Ministério da Saúde, responsável também pela distribuição às SES e às SMS de capitais e municípios com mais de 500 mil habitantes. A programação, por sua vez, passou a ser responsabilidade do Departamento de Assistência Farmacêutica e Insumos Estratégicos (DAF/MS)12.

A execução de uma programação de medicamentos qualificada depende da disponibilização de informações fidedignas, provenientes de cuidadosa análise da situação de saúde13. Com a finalidade de garantir a disponibilidade de medicamentos nas quantidades adequadas e no tempo oportuno para atender as necessidades da população, essa atividade possui impacto direto sobre o nível de abastecimento e o acesso a tratamentos nos serviços de saúde13. O processo de expansão vivenciado pelo PNCT, porém, comprometia o conhecimento da real demanda da RNTT por apoio medicamentoso, tornando a programação dos medicamentos para cessação do tabagismo um grande desafio assumido pelo DAF/MS.

O presente estudo teve por objetivo descrever a experiência de construção conjunta de planejamento estratégico para a qualificação dos processos de programação dos medicamentos do PNCT, contando com a participação de gestores municipais, estaduais e federais da assistência farmacêutica, com vistas a contribuir com a promoção do acesso ao tratamento para cessação do tabagismo no SUS. 


\section{MÉTODO}

Trata-se de estudo descritivo com relato de experiência da realização de planejamento para qualificação da programação para aquisição e distribuição dos medicamentos do PNCT.

A experiência relatada empregou a metodologia do Planejamento Estratégico Situacional (PES), de Carlos Matus ${ }^{14}$, construído através das seguintes etapas: identificação, priorização e explicação do problema (momento explicativo); definição dos objetivos, operações e ações para sua resolução (momento normativo); análise da viabilidade e factibilidade dessas operações e ações (momento estratégico); e construção de indicadores para avaliação e monitoramento da implantação do plano (momento tático-operacional).

O planejamento foi iniciado no I Seminário Nacional do Componente Estratégico da Assistência Farmacêutica (CESAF), realizado em agosto de 2014. Esse evento compôs parte do momento explicativo e contou com a participação de representantes das Coordenações da Assistência Farmacêutica dos 27 Estados brasileiros e de 10 Municípios, além de 30 gestores do DAF/MS. Durante os meses de setembro e outubro de 2014, foram realizadas duas oficinas com técnicos do DAF/MS, para delineamento dos demais momentos do planejamento. Por fim, foi analisada a execução das operações e ações delineadas no plano até a conclusão de seus prazos, em dezembro de 2015.

\section{RESULTADOS}

\section{Momento Explicativo}

A primeira etapa do planejamento ocorreu durante o Seminário Nacional do CESAF de 2014, no qual gestores da assistência farmacêutica de todos os Estados, de alguns Municípios e do Ministério da Saúde puderam debater, de forma participativa, os principais desafios e perspectivas desse Componente. Os maiores problemas identificados em relação ao planejamento associavamse à dificuldade na programação de medicamentos, às poucas capacitações para farmacêuticos sobre as doenças contempladas pelo CESAF e ao baixo número de PCDTs disponíveis para essas doenças.

Em seguida, foi realizada uma oficina com oito técnicos do DAF/MS, na qual foram priorizados e explicados os principais problemas levantados durante o seminário, de forma conjunta, em conformidade com o PES. Para a priorização, foram considerados os parâmetros de magnitude, transcendência, vulnerabilidade, urgência e factibilidade, a partir da atribuição, por cada participante da oficina, de notas de 0 a 4 para cada um dos problemas.

O problema de dificuldade na programação de medicamentos do PNCT foi considerado prioritário no seminário e na oficina. Foram identificados, então, seus descritores, as evidências 
que revelam que essa situação é um problema importante no contexto de atuação. Considerando sua intensidade e dimensão, foram apontados os seguintes descritores: ausência de dados epidemiológicos fidedignos sobre a demanda por tratamento da dependência à nicotina, dificuldade de regularização do abastecimento da RNTT e ausência de ferramenta sistematizada para uma programação adequada de medicamentos.

Posteriormente, foi possível elaborar uma matriz explicativa ou fluxograma, denominado por Matus ${ }^{14}$ de espinha de peixe (Figura 1), com a finalidade de explicar e descrever o problema. A construção deste fluxograma aconteceu por meio de chuva de ideias, a partir da contribuição de todos os participantes da oficina. Foi definida "a promoção do acesso e do uso racional dos medicamentos para cessação do tabagismo" como a imagem-objetivo do plano, seguida pela identificação da causa convergente como a "ausência de planejamento estratégico anterior à expansão do PNCT". Por dificultar o adequado conhecimento das reais necessidades da RNTT, essa causa levou à consequência convergente de "redução da eficácia do PNCT".

Figura 1 - Espinha de peixe desenvolvida a partir do problema de "dificuldade na programação para aquisição e distribuição dos medicamentos do PNCT".
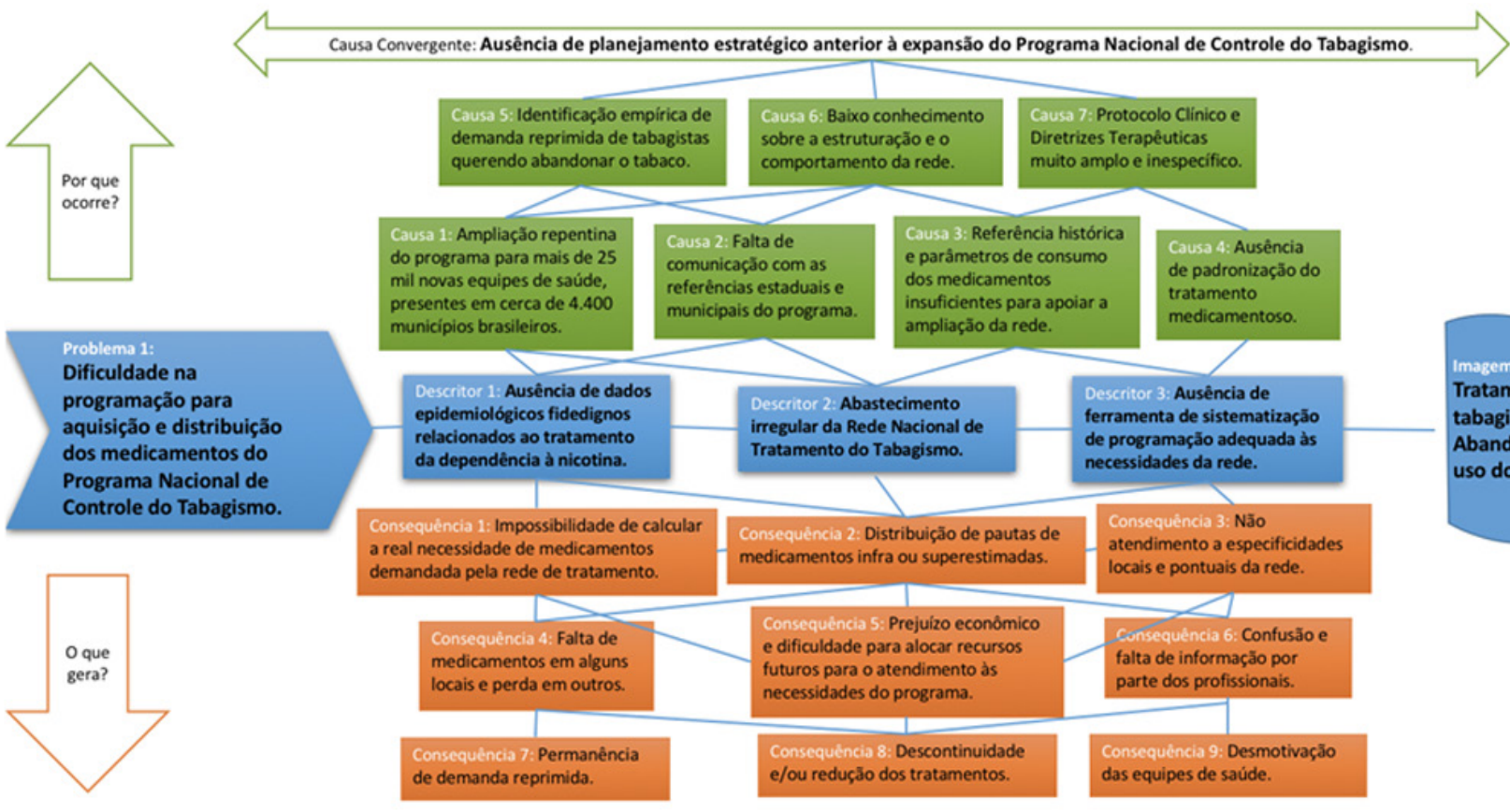

mito amplo e inespecifico.

cia histórica

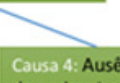

Ce padronizaçäo

tratamento

tratamento
medicamentoso.

Consequência convergente: Baixa eficácia do Programa Nacional de Controle do Tabagismo.

\section{Momento Normativo}

Após a explicação detalhada do problema, foi realizada uma segunda oficina com a participação de três técnicos do DAF/MS que trabalhavam diretamente com programação de medicamentos. Nessa reunião, foi desenvolvida a Matriz do Momento Normativo, com a intenção de direcionar às ações resolutivas para o alcance da imagem-objetivo. O objetivo geral (OG) traçado foi "desenvolver planejamento estratégico para promover a expansão racional da RNTT e

ISSN 1982-8829 Tempus, actas de saúde colet, Brasília, 11(3), 247-258, 2017 - Epub mar, 2018 
aumentar a eficácia do PNCT". Os objetivos especificos $(O E)$, para os quais foram estabelecidas operações $(O P)$ e ações $(A)$, estão apresentados no Quadro 1.

Quadro 1 - Matriz do Momento Normativo. Brasília, 2014.

\begin{tabular}{|c|c|c|}
\hline Objetivos Específicos (OE) & Operações (OP) & Ações (A) \\
\hline \multirow{3}{*}{$\begin{array}{l}\text { 1. Aumentar o acesso ao } \\
\text { tratamento para cessação do } \\
\text { tabagismo no SUS. }\end{array}$} & $\begin{array}{l}\text { 1.1 Identificar a real demanda por } \\
\text { tratamento. }\end{array}$ & $\begin{array}{l}\text { 1.1.1 Desenvolver uma ferramenta segura } \\
\text { para o adequado levantamento dos dados } \\
\text { da Rede Nacional de Tratamento. }\end{array}$ \\
\hline & \multirow{2}{*}{$\begin{array}{l}\text { 1.2 Compartilhar a gestão com as } \\
\text { áreas que coordenam o PNCT. }\end{array}$} & $\begin{array}{l}\text { 1.2.1 Redefinir as responsabilidades de } \\
\text { cada área. }\end{array}$ \\
\hline & & $\begin{array}{l}\text { 1.2.2 Levantar as informações e recursos } \\
\text { necessários. }\end{array}$ \\
\hline \multirow{3}{*}{$\begin{array}{l}\text { 2. Regularizar a } \\
\text { disponibilização de tratamento } \\
\text { medicamentoso para cessação } \\
\text { do tabagismo. }\end{array}$} & \multirow{3}{*}{$\begin{array}{l}\text { 2.1 Aperfeiçoar o processo de } \\
\text { programação para aquisição e } \\
\text { distribuição dos medicamentos. }\end{array}$} & $\begin{array}{l}\text { 2.1.1 Desenvolver uma ferramenta segura } \\
\text { para o adequado levantamento da demanda } \\
\text { por tratamento medicamentoso. }\end{array}$ \\
\hline & & $\begin{array}{l}\text { 2.1.2 Validar os parâmetros de consumo de } \\
\text { cada medicamento. }\end{array}$ \\
\hline & & $\begin{array}{l}\text { 2.1.3 Aprimorar e agilizar os processos de } \\
\text { aquisição e distribuição dos medicamentos. }\end{array}$ \\
\hline \multirow{4}{*}{$\begin{array}{l}\text { 3. Motivar as equipes e os } \\
\text { profissionais de saúde da rede } \\
\text { assistencial. }\end{array}$} & \multirow{2}{*}{$\begin{array}{l}\text { 3.1 Fortalecer o fluxo de } \\
\text { comunicação com as referências } \\
\text { dos Estados, Municípios e equipes } \\
\text { de saúde do PNCT. }\end{array}$} & $\begin{array}{l}\text { 3.1.1 Ampliar o acesso às informações } \\
\text { relacionadas ao PNCT. }\end{array}$ \\
\hline & & $\begin{array}{l}\text { 3.1.2 Compartilhar diretrizes e orientações } \\
\text { para organizar as gestões locais do PNCT. }\end{array}$ \\
\hline & \multirow{2}{*}{$\begin{array}{l}\text { 3.2 Capacitar constante e } \\
\text { continuamente os gestores e } \\
\text { profissionais de saúde atuantes. }\end{array}$} & $\begin{array}{l}\text { 3.2.1 Disponibilizar cursos de capacitação } \\
\text { e aprimoramento para profissionais da } \\
\text { Rede Nacional de Tratamento. }\end{array}$ \\
\hline & & $\begin{array}{l}\text { 3.2.2 Disponibilizar guias oficiais e } \\
\text { materiais informativos sobre o tratamento e } \\
\text { a gestão dos medicamentos. }\end{array}$ \\
\hline
\end{tabular}

\section{Momento Estratégico}

O momento estratégico também foi realizado durante a segunda oficina e teve como objetivo analisar a viabilidade e factibilidade das operações e ações propostas anteriormente, constituindo uma fase essencial para tornar o plano exequível.

A análise da viabilidade considerou o poder da esfera de atuação de decidir, executar e manter cada ação proposta. Sob a perspectiva de governabilidade direta do DAF/MS, a maior parte dessas ações se mostrou inviável, a exemplo do aprimoramento e agilização dos processos de aquisição e distribuição dos medicamentos, e da capacitação de gestores e profissionais que ofertam tratamento.

A análise da factibilidade, por sua vez, considerou os recursos necessários para a execução e manutenção das ações traçadas. Aqueles recursos necessários, mas indisponíveis, foram considerados como déficits, sendo eles principalmente recursos administrativos, humanos, 
financeiros e informacionais, além de pactuação intersetorial e conhecimento sobre as demandas da RNTT. Para cada déficit, foram formuladas atividades estratégicas, com a finalidade de superar essas limitações e criar condições para alcançar a imagem-objetivo.

Assim, diante da necessidade de pactuação intersetorial para regularizar a disponibilização dos medicamentos e aumentar o acesso ao tratamento, de forma geral, identificou-se a necessidade de sensibilizar áreas - como o INCA, o setor de compras e o almoxarifado do Ministério da Saúde sobre a importância do planejamento estratégico para a otimização da gestão do PNCT. Da mesma forma, para se ampliar o acesso às informações relacionadas à gestão do programa e fortalecer o fluxo de comunicação com os gestores locais, necessitava-se antes pactuar com estas áreas os recursos humanos e financeiros necessários para sua execução. Adicionalmente, para disponibilizar cursos e guias para aprimoramento dos profissionais da RNTT, capacitando-os para a oferta de tratamento, necessitava-se antes estimular e apoiar a produção de materiais informativos junto às áreas técnicas competentes.

\section{Momento Tático-Operacional}

A fim de acompanhar a execução do plano em construção, fez-se necessário monitorar sua implementação e avaliar seu impacto sobre a organização do serviço. Para isso, foram estabelecidos também na segunda oficina indicadores de monitoramento e avaliação, que consideravam parâmetros quantitativos ou qualitativos e detalhavam se os objetivos da proposta em elaboração estavam sendo bem conduzidos ou se foram alcançados.

Para cada operação foi criado um indicador de avaliação, cuja periodicidade da coleta de informações remetia à de ocorrência das respectivas operações, sendo de periodicidade única, trimestral ou anual. Foram descritas também as fontes de obtenção ou verificação dos dados correspondentes e levantados os recursos financeiros necessários, os principais atores e parceiros responsáveis e os prazos estimados para execução.

Como avaliação da implementação do plano, verificou-se sucesso na realização das operações cujos indicadores eram: demanda de tabagistas querendo realizar tratamento para cessação identificada; situação de abastecimento da RNTT levantada; e contato com gestores quanto às demandas da programação de medicamentos. Essas operações tinham periodicidade trimestral e prazos até abril de 2015, além de ter o DAF/MS como ator principal.

Por outro lado, não houve sucesso na realização das operações cujos indicadores eram: gestão do PNCT alinhada e compartilhada; e novos cursos de capacitação disponíveis para os profissionais da rede assistencial. Essas operações tinham prazo até abril de 2016 e exigiam mobilização de recurso financeiro e engajamento direto de outros atores, além do DAF/MS.

\section{DISCUSSÃO}

ISSN 1982-8829 Tempus, actas de saúde colet, Brasília, 11(3), 247-258, 2017 - Epub mar, 2018 
Apesar dos significativos avanços junto ao enfrentamento do tabagismo no Brasil, 21,9 milhões de brasileiros ainda consomem produtos derivados de tabaco, responsáveis por causar cerca de 130 mil mortes no país anualmente ${ }^{4,8}$. Muito ainda precisa ser feito para minimizar danos como esses à saúde da população brasileira. Pela efetividade reconhecida, o tratamento para cessação do tabagismo faz-se essencial nesse contexto, devendo ser ofertado aos usuários tabagistas em amplitude e qualidade na rede do SUS.-

O desenvolvimento de um planejamento estratégico para qualificação da programação dos medicamentos do PNCT se mostrou fundamental para a eficácia geral do tratamento para cessação ofertado no Brasil. A considerar seu impacto direto sobre o abastecimento e o acesso a tratamentos, a atividade de programação deve ser planejada de forma efetiva e participativa, e em consonância com o contexto de atuação e as reais necessidades da rede assistencial. Com a assistência farmacêutica assumindo essa responsabilidade, pretendia-se otimizar os processos de aquisição e distribuição, de forma a evitar a perda de medicamentos ou a descontinuidade de tratamentos, assegurando o adequado abastecimento da RNTT.

A utilização da metodologia do PES neste processo possibilitou analisar criticamente a realidade da rede, reconhecendo seu comportamento dinâmico e o fato de que as formas de entender as situações são múltiplas. Ao propor diálogos e reflexões permanentes por meio das oficinas, esse modelo também permitiu acompanhar a execução das intervenções delineadas e desenvolver estratégias contínuas, a fim de se obter um plano eficaz e viável do ponto de vista organizacional, operacional e de sustentabilidade.

As contribuições obtidas durante o Seminário Nacional foram importantes à medida que pautaram problemas vivenciados não somente pela gestão federal, mas também pelos Estados e Municípios, possibilitando desenvolver estratégias adequadas às necessidades e à governabilidade de cada nível de gestão. As oficinas, por sua vez, contaram com as contribuições de especialistas diretamente inseridos no contexto de atuação e agentes motivados a superar as amarras inerentes a ele.

Como resultado desse delineamento, foi possível identificar operações e ações capazes de gerar avanços em direção à promoção do acesso e do uso racional dos medicamentos do PNCT. Dentre elas estão: desenvolvimento de ferramenta segura para o levantamento dos dados da rede, incluindo a demanda por tratamento medicamentoso; redefinição das responsabilidades de cada área envolvida com a gestão do PNCT; fortalecimento do fluxo de comunicação com gestores locais; ampliação do acesso a informações gerenciais; e aprimoramento e agilização dos processos de aquisição e distribuição dos medicamentos.

Todavia, muitas dessas intervenções envolviamobstáculos quehistoricamente inviabilizavam

ISSN 1982-8829 Tempus, actas de saúde colet, Brasília, 11(3), 247-258, 2017 - Epub mar, 2018 
sua execução, sob a perspectiva do DAF/MS, seja pela ausência de poder para decidir, executar e manter as ações propostas, seja pela impossibilidade de mobilização de recursos para tanto. Ao contrário do que se esperaria, porém, recursos de ordem financeira não representaram as maiores limitações para a otimização desses processos. Esses déficits se relacionavam especialmente à maior ciência sobre as reais necessidades da RNTT e à necessidade de maior articulação entre as áreas diretamente relacionadas à gestão do PNCT, dentro do próprio Ministério da Saúde e também junto aos Estados e Municípios.

A fim de superar essas limitações e tornar o plano viável e factível, foram traçadas estratégias para sensibilizar e conseguir a cooperação das diferentes áreas gestoras do PNCT, assim como para pactuar os recursos financeiros e humanos necessários para a execução das respectivas proposições e para estimular a disponibilização de materiais informativos e apoio técnico como referenciais para a RNTT. A adoção dessas estratégias tornava o plano exequível do ponto de vista político, técnico e operacional, dando condições para transformar em realidade suas ações e, assim, o objetivo de promover o acesso e o uso racional dos medicamentos para cessação do tabagismo no SUS.

Alguns dos indicadores traçados para o monitoramento e a avaliação das intervenções sobre o serviço envolveram a utilização do formulário FormSUS-PNCT, considerado como ação-chave para viabilizar o levantamento das demandas da rede por tratamento. Essa ferramenta promovia ainda o permanente contato entre os diferentes níveis de gestão em relação às demandas de programação. Outros indicadores consideraram também o nível de abastecimento e a capacitação dos profissionais da RNTT.

As ações que puderam ser implementadas com sucesso foram aquelas que o DAF/MS pôde diretamente decidir, executar e manter. Através do uso da ferramenta FormSUS-PNCT, o nível federal da assistência farmacêutica fortaleceu o fluxo de comunicação com gestores locais e passou a dispor de dados de demanda disponibilizados pela própria RNTT. Adicionalmente, ao considerar esses dados na metodologia de programação, os processos anuais de aquisição e trimestrais de distribuição dos medicamentos passaram a se aproximar das reais necessidades da RNTT. Dados recentes do DAF/MS já demonstram que a implementação dessas medidas tem gerado um efeito crescente sobre os quantitativos de medicamentos adquiridos e distribuídos, que por sua vez, têm chegado a um número maior de municípios, quando comparado com dados de anos anteriores.

Por outro lado, a efetiva regularização do abastecimento e a ampliação do acesso aos medicamentos, de forma geral, dependem da mobilização de vários outros atores. A agilização dos processos de aquisição e garantia da disponibilidade de produtos, por exemplo, depende do setor de compras do Ministério de Saúde, enquanto que sua rápida distribuição depende também de uma rede logística que envolve almoxarifados e transportadoras. Da mesma forma, a disponibilização de cursos de capacitação e de materiais informativos para os profissionais da RNTT depende da liderança e do apoio financeiro de outras áreas, como o INCA e as SES. Não obstante, os diversos 
gestores e profissionais envolvidos com a execução do programa nas secretarias e nas unidades de saúde devem assegurar a disponibilização de informações fidedignas e oportunas sobre os tratamentos conduzidos em sua rede local.

A concretização de várias das ações deste plano esteve comprometida pela falta de alinhamento e articulação entre as diferentes áreas envolvidas com a gestão do PNCT. A participação de técnicos de outros setores, além da assistência farmacêutica, durante a construção deste planejamento viria a agregar grande valor às discussões e à própria execução do plano. Essa crítica se estende ainda ao fato de que planejamentos estratégicos, como este, devem ser realizados previamente à publicação de qualquer normativa ou diretriz de um programa ou ação em saúde, como foi o caso da portaria de 2013.

\section{CONCLUSÃO}

O desenvolvimento do presente planejamento estratégico promoveu ganhos importantes para a qualificação da programação dos medicamentos do PNCT, como a estruturação de um modelo de programação para aquisição e distribuição, e o fortalecimento da comunicação e da integração dos diferentes níveis de gestão da assistência farmacêutica. Entretanto, a efetiva regularização e ampliação dos tratamentos para cessação do tabagismo no SUS dependem do comprometimento e da ação conjunta de todas as áreas gestoras e executoras do PNCT. A impossibilidade de execução das ações que envolviam o engajamento de diferentes setores, além da assistência farmacêutica, evidencia a necessidade de forte sensibilização e de participação ativa de outros atores. Somente através da mobilização, articulação e engajamento desses gestores, e de um financiamento sustentável, é possível viabilizar as intervenções necessárias para a regularização e a ampliação da oferta de tratamentos para cessação do tabagismo no SUS.

\section{REFERÊNCIAS BIBLIOGRÁFICAS}

1. Organização Mundial da Saúde (OMS). Classificação Estatística Internacional de Doenças e Problemas Relacionados à Saúde (CID 10). $4^{\text {a }}$ Ed. - São Paulo: Editora da Universidade de São Paulo, 1997.

2. Organização Mundial da Saúde (OMS). Report on the Global tobacco epidemic, 2013. Enforcing bans on tobacco advertising, promotion and sponsorship. Geneva: WHO; 2013.

3. Organização Mundial da Saúde (OMS). Global Status Report on Noncommunicable Diseases, 2010. Geneva: WHO; 2011.

4. ACT (Aliança de Controle de Tabagismo). Relatório final: Carga das doenças tabaco relacionadas para o Brasil. Rio de Janeiro: ACT; 2012.

5. Instituto Nacional do Câncer (INCA). Programa Nacional de Controle do Tabagismo. Disponível em: http://

ISSN 1982-8829 Tempus, actas de saúde colet, Brasília, 11(3), 247-258, 2017 - Epub mar, 2018 
www2.inca.gov.br/wps/wcm/connect/acoes_programas/site/home/nobrasil/programa-nacional-controle-tabagismo/ programa-nacional - Acesso em: 11 de Fevereiro de 2016.

6. Malta, DC; Oliveira, TP; Luz, M et al. Tendências de indicadores de tabagismo nas capitais brasileiras, 2006 a 2013. Ciência Saúde Coletiva, vol. 20, n. 3. 2015. P. 631-640.

7. Ministério do Planejamento, Orçamento e Gestão; Instituto Brasileiro de Geografia e Estatística. Pesquisa Nacional por Amostra de Domicílios - Tabagismo 2008. Rio de Janeiro: IBGE; 2009.

8. Ministério do Planejamento, Orçamento e Gestão; Instituto Brasileiro de Geografia e Estatística; Ministério da Saúde; Fundação Oswald Cruz. Pesquisa Nacional de Saúde 2013: percepção do estado de saúde, estilo de vida e doenças crônicas. Brasília: IBGE; 2014.

9. Fiore, MC; Jaén, CR; Baker, TB et al. Treating Tobacco Use and Dependence. Clinical Practice Guideline. U. S. Department of Health and Humans Services, Public Health Service. 2008.

10. Ministério da Saúde. Portaria SAS/MS No 442, de 13 de Agosto de 2004. ANEXO II: Protocolo Clínico e Diretrizes Terapêuticas da Dependência à Nicotina. Diário Oficial da União, de 17 de agosto de 2004, p. 62.

11. Ministério da Saúde. Secretaria de Ciência, Tecnologia e Insumos Estratégicos. Departamento de Assistência Farmacêutica e Insumos Estratégicos. Relação Nacional de Medicamentos Essenciais - RENAME 2014. $9^{a}$ ed. Brasília: Ministério da Saúde; 2015.

12. Ministério da Saúde. Portaria GM/MS N ${ }^{\circ}$ 571, de 5 de Abril de 2013. Atualiza as diretrizes de cuidado à pessoa tabagista no âmbito da Rede de Atenção à Saúde das Pessoas com Doenças Crônicas no Sistema Único de Saúde e dá outras providências. Diário Oficial da União, de 8 de Abril de 2013, p. 56 e 57.

13. Marin, N; Luiza, VL; Castro, CG; Santos, SM. Assistência farmacêutica para gerentes municipais. Rio de Janeiro: OPAS/OMS, 2003. P. 155.

14. Matus, C. Política, Planejamento e Governo. Brasília: Instituto de Pesquisa Aplicada; 1993.

Relato apresentado em 03/05/2016

Relato aprovado em 14/03/2018

Relato publicado no sistema em 16/04/2018

ISSN 1982-8829 Tempus, actas de saúde colet, Brasília, 11(3), 247-258, 2017 - Epub mar, 2018 